\title{
Structural Basis for Shikimate-Binding Specificity of Helicobacter pylori Shikimate Kinase
}

\author{
Wen-Chi Cheng, Yu-Ning Chang, and Wen-Ching Wang* \\ Institute of Molecular and Cellular Biology and Department of Life Sciences, National Tsing Hua University, \\ Hsinchu, Taiwan
}

Received 16 June 2005/Accepted 2 September 2005

\begin{abstract}
Shikimate kinase (EC 2.7.1.71) catalyzes the specific phosphorylation of the 3-hydroxyl group of shikimic acid in the presence of ATP. As the fifth key step in the shikimate pathway for aromatic amino acid biosynthesis in bacteria, fungi, and plants, but not mammals, shikimate kinase represents an attractive target for the development of new antimicrobial agents, herbicides, and antiparasitic agents. Here, we report the 1.8-Å crystal structure of Helicobacter pylori shikimate kinase (HpSK). The crystal structure shows a three-layer alpha/beta fold consisting of a central sheet of five parallel $\beta$-strands flanked by seven $\alpha$-helices. An HpSK-shikimate-PO complex was also determined and refined to $2.3 \AA$, revealing induced-fit movement from an open to a closed form on substrate binding. Shikimate is located above a short $3_{10}$ helix formed by a strictly conserved motif (GGGXV) after $\beta_{3}$. Moreover, several highly conserved charged residues including Asp33 (in a conserved DT/SD motif), Arg57, and Arg132 (interacting with shikimate) are identified, guiding the development of novel inhibitors of shikimate kinase.
\end{abstract}

Helicobacter pylori, a gram-negative spiral bacterium, inhabits the gastric mucosa of humans, in which it may persist for a lifetime (26). The colonization of this unique ecological niche in approximately one-half of the human population makes it one of the most successful pathogens known to mankind. Enduring infection by $H$. pylori provokes active gastritis, alters gastric physiology, and may subsequently lead to peptic ulcer, atrophic gastritis, or even gastric adenocarcinoma. It is also recognized in the etiology of low-grade B-cell lymphoma (26, $35)$.

The presence of $H$. pylori infection in patients with moresevere diseases is an indication for eradication therapy. The infection, however, proves to be difficult to cure; at least two high-dose antibiotics plus a proton pump inhibitor, twice daily for a 7- to 10-day period, are required to achieve high efficacy $(21,25)$. Even more worrying, there is increasing emergence of resistant isolates that impede the cure rates $(11,14,28)$, as seen for other bacteria including Mycobacterium tuberculosis (20). The development of novel drugs for resistant infections is thus needed for more effective control of these diseases in the future.

Of particular interest from a drug development perspective, H. pylori and M. tuberculosis share seven common enzymatic components of the shikimate pathway $(1,8)$, which links metabolism of carbohydrates to biosynthesis of chorismate, a precursor of aromatic amino acids and many other aromatic compounds. Additionally, the shikimate pathway is specifically present in microorganisms, apicomplexan parasites, and plants but absent in animals $(13,38)$. Thus, enzymes involved in this pathway provide attractive targets for development of nontoxic antimicrobial compounds, herbicides, and antiparasitic agents $(5,37)$. 5-Enolpyruvylshikimate 3-phosphate (EPSP) synthase,

\footnotetext{
* Corresponding author. Mailing address: Department of Life Sciences, National Tsing Hua University, Hsinchu, Taiwan 300. Phone: 886-3-5742766. Fax: 886-3-5742766. E-mail: wcwang@life.nthu.edu.tw.
}

which catalyzes the sixth step in the pathway, has been successfully targeted with glyphosate, one of the world's bestselling herbicides (43). Similarly, knockout mutations of aroA, which encodes EPSP synthase, were found to lead to attenuation of bacterial virulence, supporting the utility of this targeting approach (15). Furthermore, fluorinated analogues of shikimate blocked the growth of Plasmodium falciparum in vitro, demonstrating that the shikimate pathway is a valid target for development of new broad-spectrum antimicrobial and antiparasitic agents (27).

Shikimate kinase (EC 2.7.1.71), the fifth enzyme of the pathway, catalyzes the specific phosphorylation of the 3-hydroxyl group of shikimic acid using ATP as a cosubstrate. In Escherichia coli, the shikimate kinase reaction is catalyzed by two isoforms that share $30 \%$ sequence identity: shikimate kinase I, encoded by the aroK gene, and shikimate kinase II, encoded by the aroL gene. Most bacteria, however, have only one shikimate kinase. The first structure of shikimate kinase from $\mathrm{Er}$ winia chrysanthemi (EcSK) demonstrates an alpha/beta protein with a central sheet of five parallel beta strands flanked by alpha helices, structurally belonging to the nucleoside monophosphate (NMP) kinase family (18). The determined apo EcSK and EcSK-MgADP complex structures reveal an opento-closed induced-fit movement of the enzyme upon substrate binding (19), as also observed in NMP kinases such as adenylate kinase $(9,42)$. Other determined shikimate kinase structures include Escherichia coli shikimate kinase I (39), Campylobacter jejuni shikimate kinase (CjSK) (not published; PDB code, 1VIA), Mycobacterium tuberculosis shikimate kinase (MtSK), the MtSK-MgADP complex (12), and the ternary MtSK-MgADP-shikimate complex $(7,36)$.

In this work, we present the crystal structures of $H$. pylori shikimate kinase (HpSK), in its apo form and in complex with shikimate and a phosphate ion. To our knowledge, HpSKshikimate- $\mathrm{PO}_{4}$ is the first shikimate kinase structure containing shikimate without MgADP. These structures provide shiki- 
mate-binding information as a rational basis for further investigation towards structure-guided inhibitors.

\section{MATERIALS AND METHODS}

Protein expression, purification, and characterization. The aroK gene (HP0157), encoding HpSK, was amplified from chromosomal DNA from $H$. pylori strain 26695 (44) by PCR using Pfu DNA polymerase and inserted into the pQE30 expression vector to generate pQE30-aroK. Expression of HpSK in E. coli JM109 cells transformed with pQE30-aroK was induced by $0.5 \mathrm{mM}$ IPTG (isopropyl- $\beta$-D-thiogalactopyranoside) at $16^{\circ} \mathrm{C}$. Bacterial pellets were fractionated, and soluble proteins in cytosolic fractions were collected. The expressed HpSK protein with a $\mathrm{His}_{6}$ tag was purified by immobilized-nickel ion chromatography, followed by Superdex-75 gel filtration chromatography (Pharmacia) and then analyzed by sodium dodecyl sulfate-polyacrylamide gel electrophoresis to verify the purity. The protein concentration was assayed according to the Bradford method with bovine serum albumin as a standard (3).

The shikimate kinase activity was determined by coupling the release of ADP from the shikimate kinase-catalyzed reaction to the oxidation of NADH using pyruvate kinase (EC 2.7.1.40) and lactate dehydrogenase (EC 1.1.1.27) as coupling enzymes (30). Shikimate-dependent oxidation of NADH was monitored by the decrease in $A_{340}\left(\varepsilon=6,200 \mathrm{M}^{-1} \mathrm{~cm}^{-1}\right)$. The assay was carried out at $25^{\circ} \mathrm{C}$ in a mixture containing $100 \mathrm{mM}$ Tris-HCl-KOH buffer, $\mathrm{pH} 7.5,50 \mathrm{mM} \mathrm{KCl}, 5$ $\mathrm{mM} \mathrm{MgCl}_{2}, 1.6 \mathrm{mM}$ shikimic acid, $2.5 \mathrm{mM}$ ATP, $1 \mathrm{mM}$ phosphoenolpyruvate, 0.1 mM NADH, 2.5 units of pyruvate kinase $/ \mathrm{ml}$, and 2.7 units of lactate dehydrogenase $/ \mathrm{ml}$. Kinetic parameters were obtained using nonlinear regression fitting to the Michaelis-Menten equation. The apparent $K_{m}$ values for each substrate were determined as follows: for ATP the shikimate concentration ([shikimate]) was maintained at $1.6 \mathrm{mM}$ and the [ATP] varied in the range from $0.001 \mathrm{mM}$ to $2.5 \mathrm{mM}$; for shikimate the [ATP] was maintained at $2.5 \mathrm{mM}$ and the [shikimate] varied in the range from 0.005 to $1.6 \mathrm{mM}$. Kinetic parameters were obtained by direct fitting to the hyperbolic saturation curves; the errors in the parameters were less than $5 \%$.

Crystallization and data collection. Crystallization was performed by the hanging-drop vapor diffusion method with 24-well tissue culture plates (Hampton Research) at $20^{\circ} \mathrm{C}$. One microliter of protein solution $\left(50 \mathrm{mg} \mathrm{ml}^{-1}\right)$ in $40 \mathrm{mM}$ Tris- $\mathrm{HCl}(\mathrm{pH} 7.0)-100 \mathrm{mM}$ sodium chloride was added to $1 \mu$ l reservoir solution and equilibrated against $0.5 \mathrm{ml}$ of reservoir solution. Initial crystallization conditions were screened using Crystal Screen I and II kits (Hampton Research) and Clear Strategy Screen I and II kits (Molecular Dimension), in which crystals were grown in a solution containing sodium acetate, $\mathrm{pH}$ 5.5, $0.2 \mathrm{M}$ lithium sulfate, and $25 \%$ polyethylene glycol (PEG) 2000MME. The best crystals of apo HpSK were obtained in a modified condition containing $0.2 \mathrm{M}$ lithium sulfate, $30 \%(\mathrm{wt} / \mathrm{vol})$ PEG 8000 , and $0.1 \mathrm{M}$ sodium acetate buffer $(\mathrm{pH}$ 6.5). The crystals grow as rods and reach a maximum size of about $0.8 \mathrm{~mm}$ by $0.1 \mathrm{~mm}$ by $0.1 \mathrm{~mm}$ within 5 days at $20^{\circ} \mathrm{C}$ and belong to space group $P 4_{2} 2_{1} 2$ with the following unit cell dimensions: $a=b=94.49 \AA$ and $c=39.30 \AA$. There is one HpSK molecule per asymmetric unit.

Shikimate complex crystals could not be obtained by soaking with shikimate. Instead, complex crystals were obtained by cocrystallizing with $10 \mathrm{mM}$ shikimate, $10 \mathrm{mM} \mathrm{MgCl}_{2}$, and $10 \mathrm{mM} \mathrm{ADP}$ under conditions containing lithium sulfate as the precipitant. Crystals were immediately obtained in a screening solution consisting of $1.8 \mathrm{M} \mathrm{Li}_{2} \mathrm{SO}_{4}$ and $0.1 \mathrm{M}$ sodium cacodylate buffer (pH 6.5). The crystals were shown to belong to the space group $P 6_{1}$ with the following cell dimensions: $a=b=97.29 \AA$ and $c=46.91 \AA$. There is one HpSK monomer per asymmetric unit.

Prior to data collection, crystals were dipped into Fomblin cryoprotectant oil for several seconds and then flash-frozen in a liquid nitrogen stream. Diffraction data were collected using a MSC X-Stream cryosystem and an R-AXIS IV ++ image plate system with double-mirror-focused $\mathrm{CuK} \alpha \mathrm{X}$-ray radiation generated from a Rigaku RU-300 rotating-anode generator at the Macromolecular X-ray Crystallographic Laboratory of National Tsing Hua University, Hsinchu, Taiwan. The 1.8- $\AA$ native data set for apo HpSK was collected on the BL12B2 Taiwan beamline at SPring-8, Hyogo, Japan, using an ADSC Quantum 4R chargecoupled device detector. All data sets were collected at $-150^{\circ} \mathrm{C}$ and processed with the HKL/HKL2000 suite (34). Data collection statistics are shown in Table 1.

Structure determination and refinement. The apo HpSK structure was solved by molecular replacement with the program AMoRe (33), using the structure of CjSK (PDB code, 1VIA) as the search model. Rotation and translation functions followed by the rigid body refinement procedure of AMoRe $(4,33)$ were carried out using data from 8- to 4- $\AA$ resolution and yielded one outstanding solution (fractional coordinates, $x=0.136, y=0.682$, and $z=0.078$ ). Crystallographic
TABLE 1. X-ray data and refinement statistics

\begin{tabular}{|c|c|c|}
\hline \multirow[b]{2}{*}{ Parameter } & \multicolumn{2}{|c|}{ Result for: } \\
\hline & Apo HpSK & $\begin{array}{c}\text { HpSK-shikimate- } \\
\mathrm{PO}_{4}\end{array}$ \\
\hline \multicolumn{3}{|l|}{ Data collection and phasing } \\
\hline Wavelength (A) & 1.000 & 1.5418 \\
\hline Space group & $P 4_{2} 2_{1} 2$ & $P 6_{1}$ \\
\hline Unit cell $(\AA)$ & $\begin{array}{c}a=b=94.49 \\
c=39.30\end{array}$ & $\begin{array}{c}a=b=97.29 \\
c=46.91\end{array}$ \\
\hline Resolution limit $(\AA)$ & 1.80 & 2.30 \\
\hline No. of unique reflections & 17,190 & 11,417 \\
\hline Completeness $(\%)^{a}$ & $98.9(90.1)$ & $99.7(97.9)$ \\
\hline $\operatorname{Avg} I / \sigma(I)$ & $21.5(5.2)$ & $9.2(4.2)$ \\
\hline$R_{\text {merge }}(\%)^{b}$ & $4.3(39.5)$ & $9.3(45.1)$ \\
\hline \multicolumn{3}{|l|}{ Refinement } \\
\hline Resolution range $(\AA)$ & $67-1.8$ & $85-2.3$ \\
\hline No. of protein atoms & 1,198 & 1,261 \\
\hline No. of solvent atoms & 165 & 66 \\
\hline$R$ factor $^{c}$ & 0.210 & 0.203 \\
\hline$R_{\text {free }}{ }^{d}$ & 0.266 & 0.280 \\
\hline r.m.s.d. bond length $(\AA)^{e}$ & 0.021 & 0.042 \\
\hline r.m.s.d. bond angles $\left({ }^{\circ}\right)$ & 1.99 & 3.25 \\
\hline r.m.s.d. bond torsion angle $\left({ }^{\circ}\right)$ & 6.49 & 10.27 \\
\hline \multicolumn{3}{|l|}{ Ramachandran analysis $(\%)$} \\
\hline Favored & 96.2 & 95.0 \\
\hline Allowed & 3.0 & 3.6 \\
\hline Generous & 0.8 & 1.4 \\
\hline Disallowed & 0.0 & 0.0 \\
\hline Estimated coordinate error $(\AA)$ & 0.136 & 0.236 \\
\hline
\end{tabular}

${ }^{a}$ Values in parentheses refer to statistics in the highest-resolution shell. ${ }^{b} R_{\text {merge }}=\Sigma\left|I_{\text {obs }}-\langle I\rangle\right| \Sigma I_{\text {obs }}$.

${ }^{c} R=\Sigma\left|F_{\text {obs }}-F_{\text {calc }}\right| / \Sigma F_{\text {obs }}$, where $F_{\text {obs }}$ and $F_{\text {calc }}$ are the observed and calculated structure factor amplitudes, respectively.

${ }^{d} R_{\text {free }}$ was computed using $5 \%$ of the data assigned randomly.

${ }^{e}$ r.m.s.d., root mean square deviation.

refinement was carried out using the maximum-likelihood target function embedded in program REFMAC5 (32) and coupled to ARP/wARP (22). Five percent of the reflections were randomly selected and used to compute a free- $R$ value $\left(R_{\text {free }}\right)$ for cross-validation of the model. $2 F_{o}-F_{c}$ and $F_{o}-F_{c}$ maps were produced and inspected after each cycle of refinement to revise the model manually on an interactive-graphics computer with the program $\mathrm{O}(16)$. The progress of the refinement was evaluated by the improvement in the quality of the maps as well as the reduced values for $R$ and $R_{\text {free }}$. Geometrical restraints were then applied and gradually relaxed during the refinement (2). The overall stereochemical quality of the final model was assessed by the program PROCHECK (23).

Structural comparisons. Structure comparisons with CjSK (PDB code, 1VIA), EcSK (PDB code, 1SHK), EcSK-ADP (PDB code, 2SHK), EcSK (K15M-P115L mutant)-PO ${ }_{4}$ (PDB code, 1E6C), MtSK-MgADP (PDB codes, 1L4U and 1L4Y), and MtSK-MgADP-shikimate (PDB codes, 1U8A and 1WE2) were carried out using the program LSQMAN in O (16) to superimpose $\mathrm{C} \alpha$ atoms based on the optimized alignment of the central $\beta$-sheets. Combined sequence and secondary structure alignments and figure preparation were done with the program ESPript (10). Electron density map figures were prepared with PyMOL (www.pymol.org). Structural figures were prepared with the programs MOLSCRIPT (17) and RASTER3D (29).

Atomic coordinates. The coordinates and structure factors of the HpSK and HpSK-shikimate- $\mathrm{PO}_{4}$ structures have been deposited in the RCSB Protein Data Bank with accession codes $1 \mathrm{ZUH}$ and 1ZUI, respectively.

\section{RESULTS AND DISCUSSION}

Expression, purification, and enzymatic analysis of HpSK. Full-length HpSK was overexpressed in E. coli JM109(pQE30aroK). After purification by affinity chromatography, a major band of an apparent molecular mass of $\sim 20 \mathrm{kDa}$ was observed by sodium dodecyl sulfate-polyacrylamide gel electrophoresis 
(data not shown). The purified protein indeed possessed specific phosphoryl transfer activity toward shikimate in the presence of ATP. The apparent values for Michaelis-Menten parameters were determined as follows: $K_{m(\mathrm{MgATP})}=101 \pm 17$ $\mu \mathrm{M}, K_{m \text { (shikimate) }}=60 \pm 8 \mu \mathrm{M}, V_{\max (\mathrm{MgATP})}=26 \pm 1 \mu \mathrm{mol} /$ $\mathrm{min} / \mathrm{mg}$, and $V_{\max (\text { shikimate) }}=22 \pm 1 \mu \mathrm{mol} / \mathrm{min} / \mathrm{mg}$. The $K_{m \text { (shikimate) }}$ value is much lower than that for $\operatorname{EcSK}(310 \mu \mathrm{M})$ and that for $E$. coli shikimate kinase II $(200 \mu \mathrm{M})$ while ATP is held at a high concentration. On the other hand, E. coli shikimate kinase II has a similar value of apparent $K_{m(\mathrm{MgATP})}(160$ $\mu \mathrm{M})$, while EcSK has a much higher apparent $K_{m \text { (MgATP) }}(620$ $\mu \mathrm{M})(6,12,19)$.

Structure descriptions. The 1.8 - $\AA$-resolution electron density map of unliganded HpSK reveals one molecule per asymmetric unit. The overall dimensions of the oval-shaped molecule are $35 \AA$ by $35 \AA$ by $30 \AA$ (Fig. 1A). Three segments are omitted from the model due to negative or weak density: the $\mathrm{N}$-terminal methionine, 2 residues at the $\mathrm{C}$ terminus, and a region (residues 111 to 118 ) which connects two $\alpha$-helices and is in a flexible loop, as seen in other shikimate kinase structures $(12,18)$. The final model was refined to an $R$ value of $21.0 \%$ $\left(R_{\text {free }}=26.6 \%\right)$ (Table 1$)$. The mean B factor of protein atoms in HpSK is $27.5 \AA^{2}$.

Complex crystals were obtained by cocrystallization with HpSK, $10 \mathrm{mM}$ shikimate, and $10 \mathrm{mM}$ ADP under conditions that included $1.8 \mathrm{M} \mathrm{Li}_{2} \mathrm{SO}_{4}$ and $0.1 \mathrm{M}$ sodium cacodylate $(\mathrm{pH}$ $6.5)$. The complex structure was determined by molecular replacement methods and refined to an $R$ value of $20.3 \%\left(R_{\text {free }}\right.$ $=28.0 \%$ ) (Table 1$)$. The $2 F_{o}-F_{c}$ map defines the location and orientation of shikimate in a solvent-accessible cleft (Fig. 1). Apart from shikimate, the pocket includes a piece of strong density $(>2.5 \sigma)$ that can be modeled as a phosphate ion or a sulfate ion. Since a phosphate ion has been identified in the EcSK K15M mutant (PDB code: 1E6C) at a similar position, we modeled this density as a phosphate moiety. No other signal corresponding to MgADP was observed. The mean B factor of protein atoms in the complex is higher than that of the apo structure (50.3 versus $27.5 \AA^{2}$, respectively). Notably, high B factors are seen for residues 99 to 110 and 113 to 122 , which display significant conformational changes upon substrate binding, similar to those observed in the complex form of EcSK or $\operatorname{MtSK}(7,12,18,36)$. The overall root mean square deviation in $\mathrm{C} \alpha$ atom positions between the superimposed $\mathrm{HpSK}$ and HpSK-shikimate- $\mathrm{PO}_{4}$ structures is $0.68 \AA$.

The HpSK fold consists of three domains characteristic of members in the NMP kinase family (Fig. 1A): (i) the CORE domain (residues 1 to 31,61 to 108 , and 124 to 162), (ii) the shikimate-binding (SB) domain (residues 32 to 60; corresponding to NMP-binding domain in adenylate kinases), and (iii) the LID region (residues 109 to 123$)(31,40-42,45)$. A three-layer $\alpha-\beta-\alpha$ fold is formed, in which the central parallel sheet (strand order: 23145) of the CORE domain is flanked by $7 \alpha$-helices.

Conformational changes: apo versus liganded structures. In contrast to the apo structure, the liganded structure has an ordered "lid" loop that closes the binding pocket. The LID region consists of a long loop (residues 109 to 123 ), which has 8 residues (111 to 118 ) missing in the apo structure. In the liganded form, it is mostly defined except for 2 residues (111 and 112). Superposition of apo and complex HpSK structures reveals the essentially overlapped central $\beta$-sheets, compared
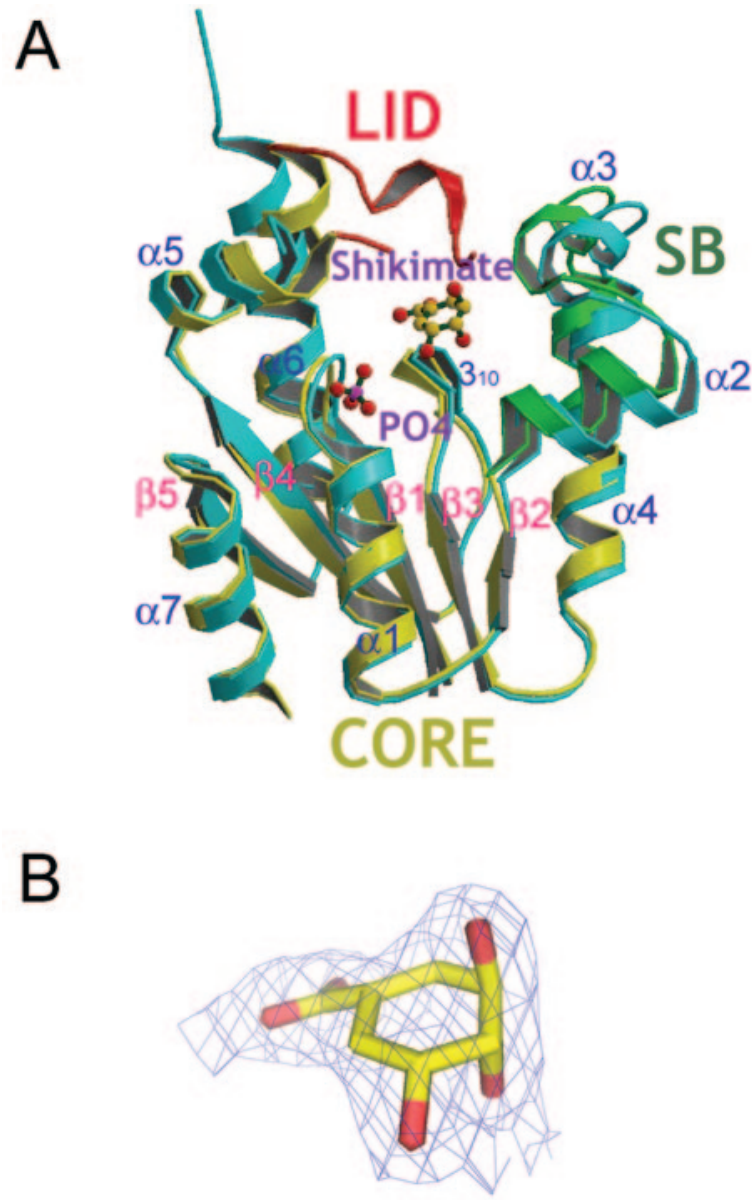

FIG. 1. Structure of HpSK. (A) Superimposed structures of HpSK and the HpSK-shikimate- $\mathrm{PO}_{4}$ complex. The $\mathrm{C} \alpha$ traces of the apo $\mathrm{HpSK}$ structure are in cyan. The HpSK-shikimate- $\mathrm{PO}_{4}$ complex is depicted based on three domains: CORE domain (yellow), SB domain (green), and LID region (red). The ball-and-stick model of shikimate and phosphate is shown in dark green. The carbon, oxygen, and phosphorus atoms are in yellow, red, and pink, respectively. (B) $2 F_{o}-F_{c}$ electron density map of the HpSK-shikimate- $\mathrm{PO}_{4}$ complex around shikimate, contoured at the 1.1- $\sigma$ level.

with the apparent deviation seen in the SB and LID domains (Fig. 1A). Flexibility of these two regions is indicated by the much higher B-factor value in the shikimate-containing structure, particularly the LID region (data not shown). The most striking deviation in the distance between $\mathrm{C} \alpha$ pairs is seen in residues 106 to 110 and 119 to 126 of the LID domain, particularly Leu119 (17.08 ^). The conformational changes seen in the complex structure are a characteristic feature of induced domain closure under substrate binding that is found among shikimate kinases and adenylate kinases $(18,42)$.

The shikimate-binding pocket. Shikimate is bound in an enclosed pocket surrounded by the C-terminal ends of the central $\beta$-sheet in the various shikimate kinases. Several conserved motifs are found (Fig. 2): (i) the segment after $\beta_{1}$ that contains Walker A motif GXXGXGKT/S (residues 8 to 15); (ii) the DT/SD motif (residues 31 to 33 ) after $\beta_{2}$, in which Asp33 is essential to interact with $\mathrm{O}-11$ and $\mathrm{O}-12$ of the shikimate; (iii) the GGGXV segment (residues 79 to 83) containing 


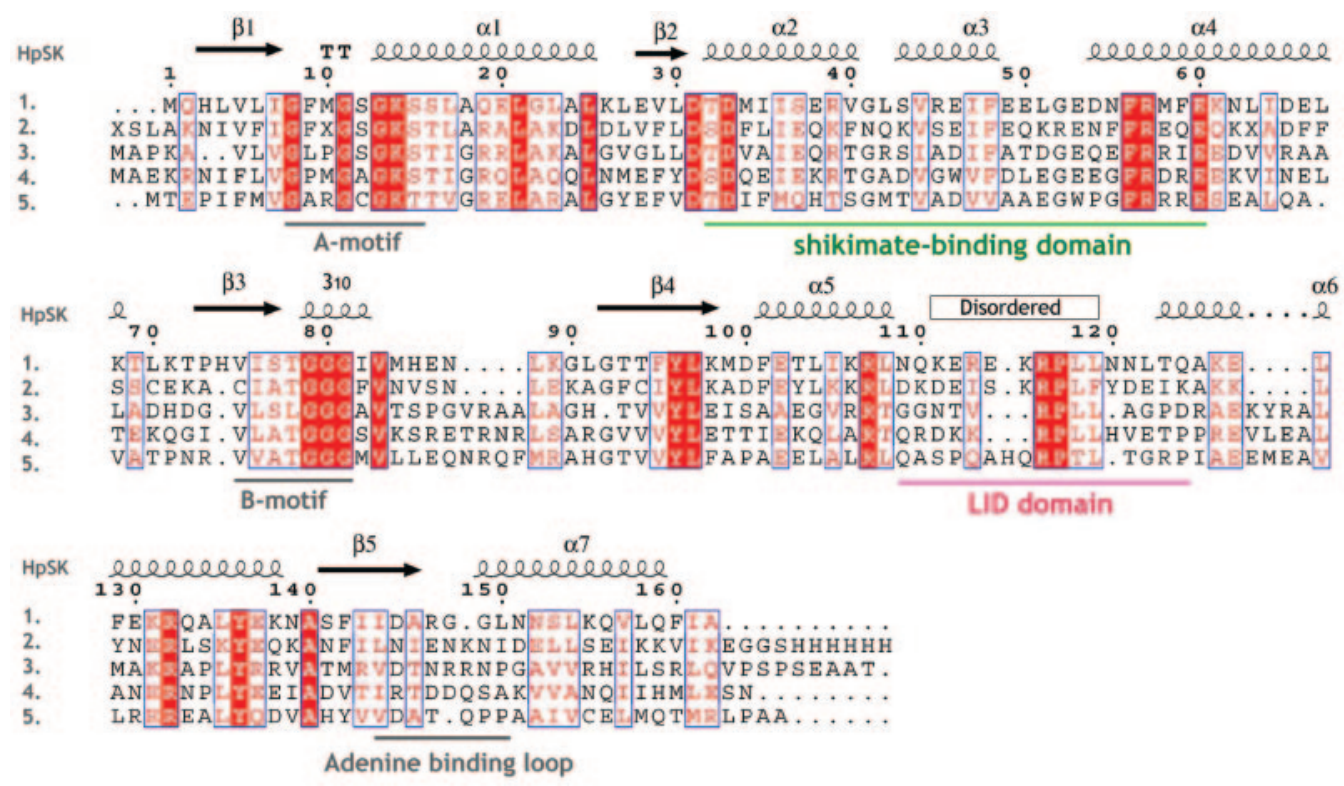

FIG. 2. Structure-based alignment of HpSK with other shikimate kinases. 1, Helicobacter pylori shikimate kinase; 2, Campylobacter jejuni shikimate kinase; 3, Mycobacterium tuberculosis shikimate kinase; 4, E. coli shikimate kinase I; 5, Erwinia chrysanthemi shikimate kinase. The secondary structural elements are shown above the sequence. Red boxes enclose conserved positions, and open boxes show positions with homologous residues. The shikimate-binding domain and LID domain are indicated. The Walker A motif (P loop), Walker B motif, and adenine-binding loop are also underlined.

a $3_{10}$ helix after $\beta_{3}$. It is noted that the consensus shikimate kinase Walker B motif (ZZZTGGG) (24) of HpSK (residues 75 to 81 ) partly overlaps the GGGXV segment.

In the HpSK structure, the Walker A motif (GFMGSGKS) (residues 8 to 15 ) after $\beta_{1}$ forms a hole to accommodate the phosphate ion with numerous interactions, in accord with its proposed role in binding to the $\beta$-phosphate of the nucleotide (45). In the Walker B motif of HpSK (VISTGGG) (residues 75 to 81 ), the conserved Gly80 has an orientation that may interact with the $\gamma$-phosphate of a bound ATP. Shikimate kinase Walker B motifs lack an Asp residue involved in coordinating the active site $\mathrm{Mg}^{2+}$, and, instead, shikimate kinases have the DT/SD motif at residues 31 to $33(24,36)$.

Gly79, which is in both the GGGXV segment and Walker B motif, has two direct contacts with O-11 of shikimate. In total, the glycines in the GGGXV segment (Gly79, Gly80, and Gly81) make 10 interactions with shikimate $(<3.8 \AA)$, including a hydrogen bond with the peptide $\mathrm{N}$ atom of Gly80 (Table $2)$. The other conserved residue in the GGGXV segment (Val83) interacts with Gly81 (Gly81 [O]-Val83 [N]; $2.90 \AA$ ) to form a stable $3_{10}$ helix to support shikimate binding.

Additionally, residues from the SB, LID, and CORE domains interact with shikimate: (i) Asp33, Val44, Phe48, and Arg57 from the SB domain; (ii) Glu114 and Arg116 from the LID region, which are missing in the unliganded form, and (iii) Met10 and Arg132 from CORE (Fig. 3A).

Residues interacting with functional groups in shikimate can be considered to form three subsites on the protein: (i) $C_{X}$, which contacts a carboxyl moiety of shikimate (C-1, O-2, O-3, and $\mathrm{C}-4$ ); (ii) $\mathrm{O}_{\mathrm{CORE}}$, which interacts with two hydroxyl groups of shikimate (C-8, C-9, C-10, O-11, and O-12); and (iii) $\mathrm{O}_{\text {LID }}$, which interacts with a trans hydroxyl group of shikimate (C-5, C-6, and O-7) (Fig. 3B and Table 2).
In the $\mathrm{C}_{\mathrm{X}}$ site, the carboxyl moiety of shikimate forms 17 interactions $(<3.8 \AA)$ with nearby residues including a hydrogen bond with Arg57 from SB and 2 with Arg132 from CORE. The O-3 atom in shikimate also binds to a water molecule bridging to Met10 (Wat3 [O]-Met10 N; $3.20 \AA$ ) from the CORE domain.

In the $\mathrm{O}_{\mathrm{CORE}}$ subsite, apart from interactions with Gly79, Gly80, and Gly81 on the $3_{10}$ segment, there are 11 direct contacts $(<3.8 \AA)$ with nearby residues. Two contacts are found with two water molecules, bridging to Lys14 and Arg57 (shikimate [O-11]-Wat11 [O]-Lys14 [N $]$ ]; 2.92 to $2.74 \AA$; shikimate [O-12]-Wat2 [O]-Arg57 [Nๆ2]; 3.01 to $2.67 \AA$ ). Significantly, three strong interactions are formed with Asp33 of the conserved motif DT/SD after $\beta_{2}$ (Table 2).

For the $\mathrm{O}_{\text {LID }}$ portion, the trans hydroxyl group primarily interacts with Glu114 and Arg116 from the LID region, including two strong bonds. Notably, Glu114 and Arg116 are missing in the apo form, whereas they become ordered in the liganded structure and point toward the bound shikimate, closing up the binding pocket. In total, there are 47 interactions within $3.8 \AA$, including 11 hydrogen bonds. These considerable interactions together enable the flexible LID and SB domains to move toward shikimate, producing a disordered-to-ordered switch to close up the interface cleft.

Comparison of the shikimate-binding pockets between MtSK and HpSK. Since MtSK is the only other shikimate kinase structure that has been determined with a bound shikimate molecule in the binding pocket, it was of interest to compare its binding pocket with that of HpSK. The significant structural homology between MtSK and $\mathrm{HpSK}$ is notable given their relatively low sequence identity $(25 \%)$.

There are significant similarities in the $\mathrm{O}_{\mathrm{CORE}}$ and $\mathrm{C}_{\mathrm{X}}$ subsites between both enzymes. Superposition of the HpSK-shiki- 
TABLE 2. Binding of shikimate in HpSK (this work) and in MtSK (7)

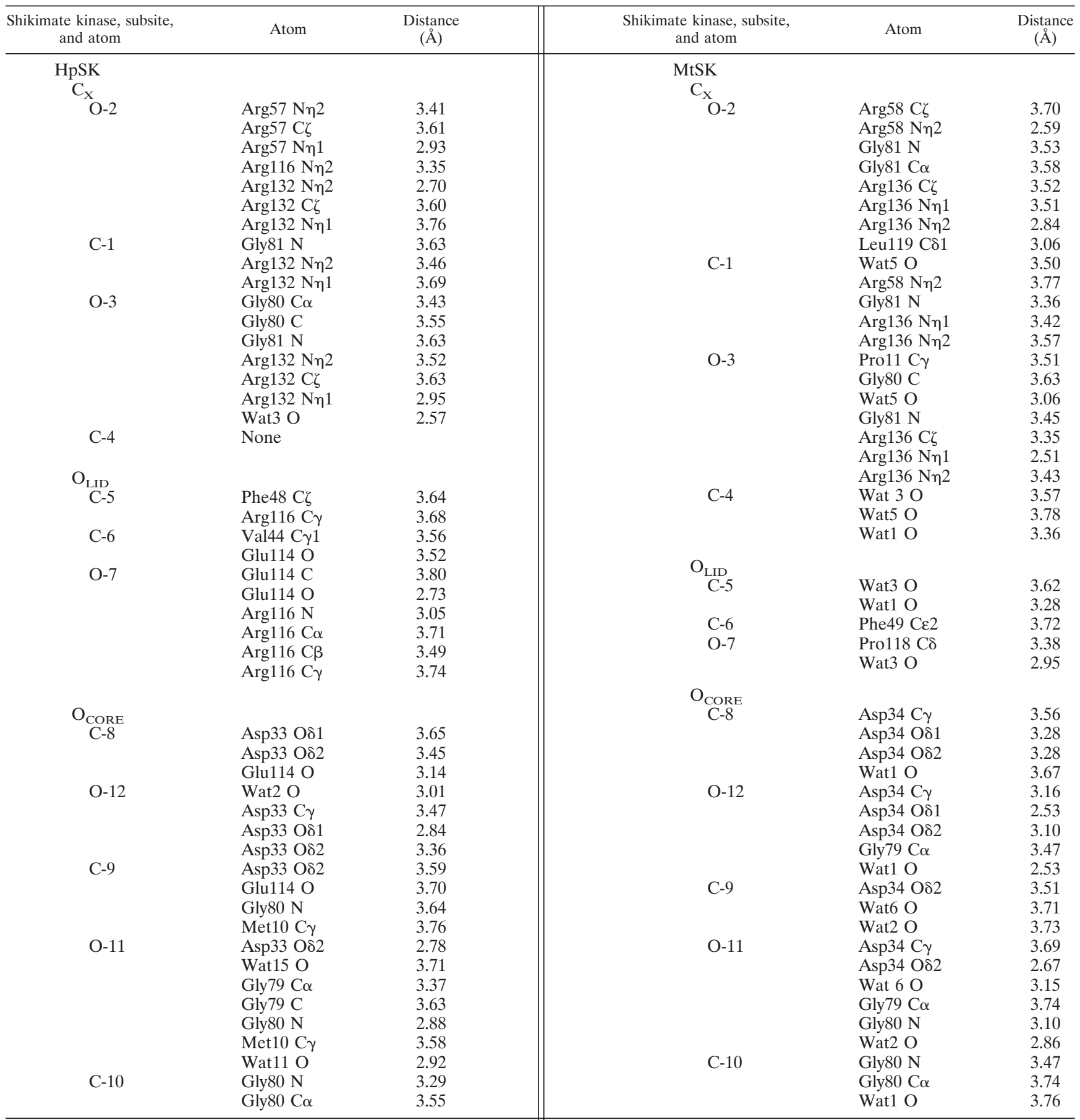

mate- $\mathrm{PO}_{4}$ and MtSK-MgADP-shikimate (7, 36) structures based on the optimized alignment of their central $\beta$-sheets shows that several residues overlap relatively well from the $\mathrm{O}_{\text {CORE }}$ and $\mathrm{C}_{\mathrm{X}}$ subsites: Asp33, Arg57, Gly79, Gly80, Gly81, and Arg132 in HpSK; Asp34, Arg58, Gly79, Gly80, Gly81, and Arg136 in MtSK (Fig. 4). It is particularly noted that Asp33 of the DT/SD motif and Gly79 Gly80 Gly81 of the GGGXV motif, which interacts extensively with shikimate, are essentially overlapped at similar positions (Table 2 and Fig. 4).
These two strictly conserved segments are thus considered shikimate-binding motifs among shikimate kinases.

In contrast, there are differences in residues from the $\mathrm{O}_{\text {LID }}$ subsite that interact with the trans hydroxyl group (O-7) of shikimate. Residues in this subsite are not conserved between the two organisms, except for Phe 48 in HpSK (corresponding to Phe49 in MtSK). Thus, in HpSK, Val44, Phe48, Glu114, and Arg116 form the $\mathrm{O}_{\text {LID }}$ subsite, whereas Phe49 and Pro118 form the corresponding subsite in MtSK. 
A
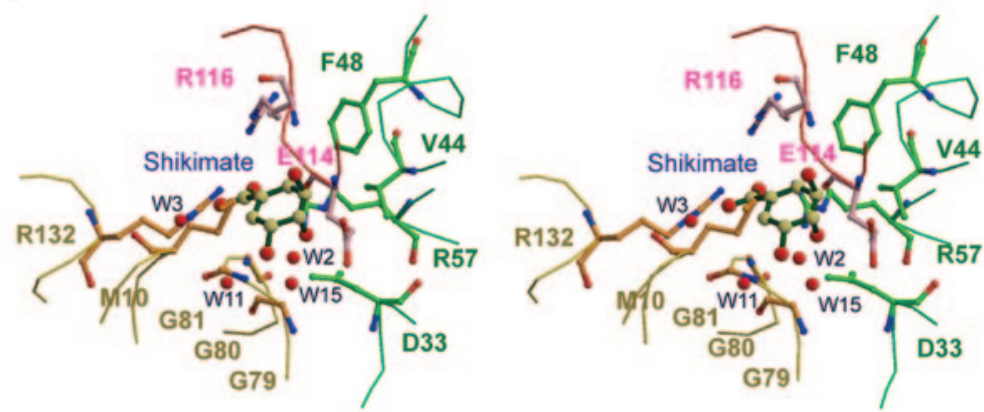

B

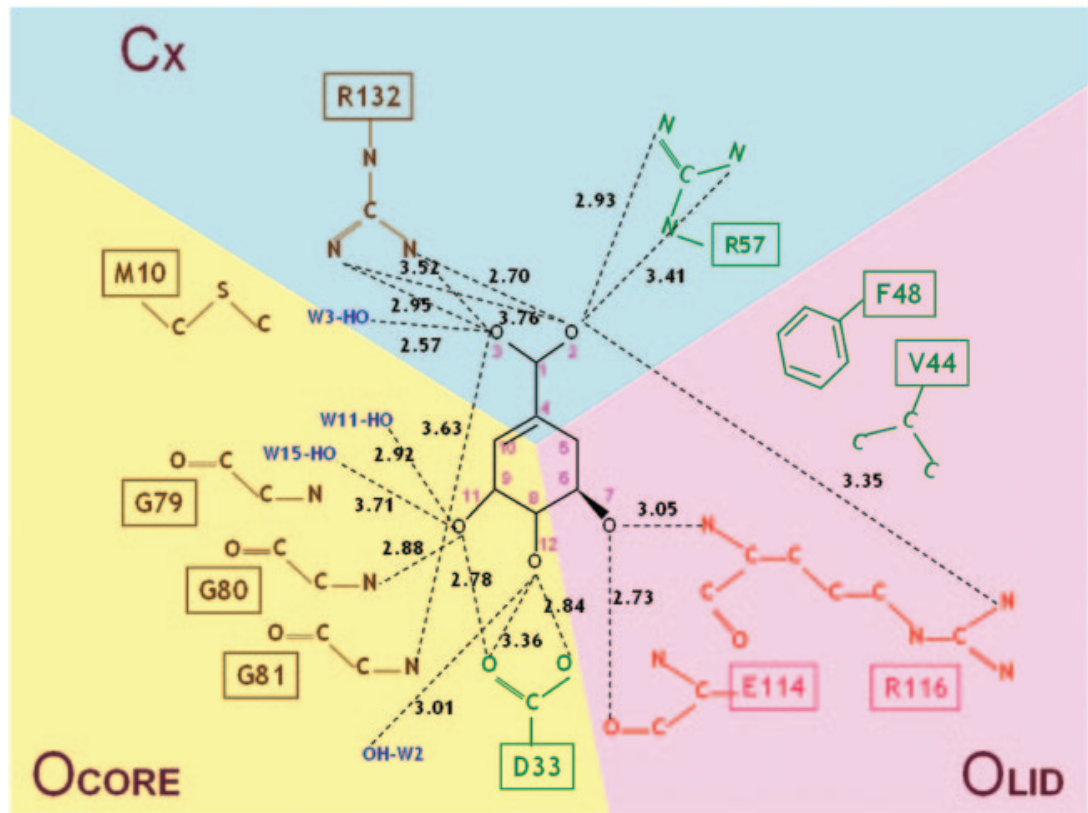

FIG. 3. The shikimate-binding pocket in the HpSK-shikimate- $\mathrm{PO}_{4}$ complex. (A) Stereoview of the shikimate binding site. Shikimate (dark green) is shown as a ball-and-stick model with carbon atoms colored white. Residues in SB (green), CORE (orange), and LID (pink) regions are shown as stick models. The C $\alpha$ traces of SB, CORE, and LID enclosing shikimate are depicted in green, yellow, and red, respectively. The oxygen and nitrogen atoms are in red and blue, respectively. (B) Schematic representation of interactions between shikimate and HpSK. The color representation of the three regions is as in panel A. Hydrogen-bonding interactions are shown as dashed lines.

The $\mathrm{O}_{\text {LID }}$ subsite in HpSK also interacts with shikimate to a greater extent than does the corresponding subsite in MtSK; there are 11 contacts $(<3.8 \AA)$ including two strong interactions with Glu114 and Arg116 in HpSK compared with only two van der Waals interactions in MtSK. One likely explanation is the presence of three additional residues in the loop of the LID domain of HpSK, enabling the 113-to-122 segment to interact more extensively with shikimate and also with residues from the SB domain located on the opposite side of the binding pocket. Thirteen direct contacts $(<3.8 \AA)$ are observed between the LID (Arg113, Glu114, and Lys115) and SB domains (Met34, Ser43, Val44, and Arg45) in the HpSK complex compared with few direct contacts (1U8A, three interactions; $1 \mathrm{WE} 2$, two interactions) in MtSK-MgADP-shikimate structure.

Superpositions with the MtSK complex also give insight into the residues that would bind to the nucleotide cofactor in
HpSK. One conserved Arg residue of the LID domain, which is involved in binding to ADP (Arg110 in MtSK), corresponds to Arg107 in HpSK. Superimposed structures show that Arg110 of MtSK and Arg107 of HpSK are situated approximately in similar positions, suggesting that Arg107 in HpSK may play a similar role in binding the cofactor. In contrast, another conserved Arg117 of MtSK deviates away from Arg116 of HpSK, whose side chain points in the opposite direction of the superimposed ADP moiety. Instead, Arg116 of HpSK interacts with the trans hydroxyl group of shikimate (above). Interestingly, the superimposed structures reveal that the side chain of a different arginine, Arg113 in HpSK, faces toward the superimposed MgADP with an $\sim 6-\AA$ distance between the $\mathrm{C}_{\alpha}$ atom of $\operatorname{Arg} 117$ in MtSK and that of Arg113 in HpSK. It is thus possible that Arg113 instead of Arg116 of HpSK is the second Arg in a conserved motif $\mathrm{R}(\mathrm{X})_{6-9} \mathrm{R}$ for P-loop kinases $(24,36)$. Upon binding to the nucleotide cofac- 

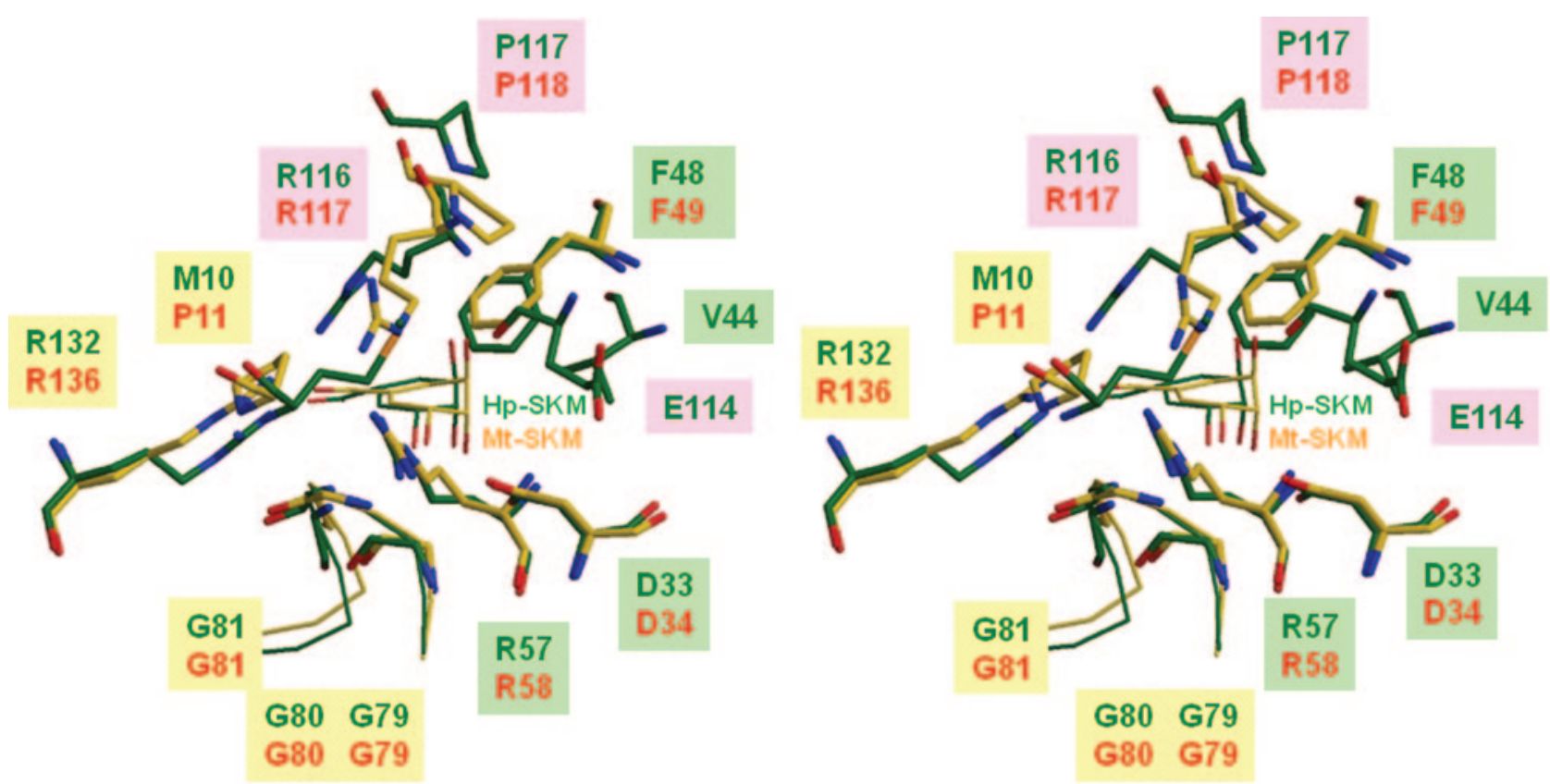

FIG. 4. Superposition of shikimate-binding residues of HpSK (green) and MtSK (yellow) complex structures. Residues involved in substrate binding are shown as stick structures (Met10, Asp33, Val44, Phe48, Arg57, Gly79, Gly80, Gly81, Glu114, Arg116, Pro117, and Arg132 in HpSK; Pro11, Asp34, Phe49, Arg58, Gly79, Gly80, Gly81, Arg117, Pro118, and Arg136 in MtSK). The bound shikimate (SKM) in HpSK (green) or in MtSK (yellow) is shown as thin sticks.

tor, additional domain closure would be expected to take place, as seen in the MtSK-MgADP-shikimate structure (discussed below), allowing Arg113 bind to the cofactor. The key residues involved in binding to shikimate or ATP may thus vary in the LID domains of various shikimate kinases owing to the higher divergence of this region, which may also contribute to the variation in kinetic parameters.

Binding of shikimate contributes to induced-fit conformational changes. It is likely that the binding of shikimate rather than the phosphate ion leads to the conformational changes observed in the ternary $\mathrm{HpSK}$-shikimate- $\mathrm{PO}_{4}$ complex. First, no direct contacts from the LID domain to the phosphate ion are observed in the ternary $\mathrm{HpSK}$-shikimate- $\mathrm{PO}_{4}$ structure. Additionally, in this structure, and in the K15M mutant EcSK$\mathrm{PO}_{4}$ binary complex, which is the only other structure with a bound phosphate ion (19), the phosphate is positioned similarly to the $\beta$-phosphate of ADP in other shikimate kinase complexes and is surrounded only by the Walker A GXXGX GKT/S segment after $\beta_{1}$. The binding of a single phosphate ion is thus unlikely to result in a significant structural change in the LID domain, which it does not contact. Instead, such changes are likely to result from shikimate binding.

Similar large induced conformational changes have also been seen in EcSK and in MtSK structures upon MgADP binding $(12,18,19)$. Analysis of superimposed apo and complex structures suggests that those motions are caused by two independent hinges with joints at similar positions upon binding to either MgADP or shikimate. This suggests that the binding of either ligand into the pocket of shikimate kinases can independently induce the closure involving the SB and LID domains.

As shown by two recently solved ternary MtSK-MgADP- shikimate structures, there is an even tighter pocket in the ternary structure compared with the MtSK-MgADP binary structure $(7,36)$. There are shifts in residues from the LID and SB domains, which explain the additional closure involving both domains. Moreover, the induced-fit conformational change is also governed by the SB and LID hinges. Evidence from circular dichroism spectroscopic analysis also reveals synergy in substrate binding (19). Taken together, these results suggest synergistic conformational change when both shikimate and ATP bind to the pocket, yielding a completely sealed form to exclude additional solvent molecules for the catalytic reaction to take place.

In conclusion, we have expressed and characterized $H$. pylori shikimate kinase. We also present the HpSK structure in its apo form and in complex with shikimate and a phosphate ion. The structures reveal that HpSK has an overall architecture characteristic of the NMP kinase superfamily. Comparison of the apo and the shikimate-bound structures reveals an opento-closed conformational change that takes place as the substrate binds to a solvent-accessible pocket. We have identified three subsites $\left(\mathrm{C}_{\mathrm{X}}, \mathrm{O}_{\mathrm{CORE}}\right.$, and $\left.\mathrm{O}_{\text {LID }}\right)$ that interact with shikimate and $\mathrm{PO}_{4}{ }^{3-}$. Based on the structural information, Asp33, Gly79, Gly80, and Gly81 in $\mathrm{O}_{\text {CORE }}$ and Arg57 and Arg132 in $\mathrm{C}_{\mathrm{X}}$, which share significant conservation among shikimate kinases, are critical in binding to shikimate, providing a rational basis to guide the development of novel inhibitors.

\section{ACKNOWLEDGMENTS}

We acknowledge access to The Macromolecular X-ray Crystallographic Center of NTHU Instrument Center at Hsinchu, National Tsing Hua University; the BL17B2 beamline at the National Synchrotron Radiation Research Center (NSRRC), Taiwan; and the BL12B2 
Taiwan beamline at SPring-8, Japan, for data collection. We also thank the staff at NSRRC for their excellent support.

This work was supported by grants from NSC (NSC94-3112-B-007005 and NSC93-3112-B007-013).

\section{REFERENCES}

1. Alm, R. A., and T. J. Trust. 1999. Analysis of the genetic diversity of Helicobacter pylori: the tale of two genomes. J. Mol. Med. 77:834-846.

2. Anonymous. 1994. The CCP4 suite: programs for protein crystallography. Acta Crystallogr. Sect. D 50:760-763.

3. Bradford, M. M. 1976. A rapid and sensitive method for the quantitation of microgram quantities of protein utilizing the principle of protein-dye binding. Anal. Biochem. 72:248-254.

4. Castellano, E., G. Oliva, and J. Navaza. 1992. Fast rigid-body for molecular replacement techniques. J. Appl. Crystallogr. 25:281-284.

5. Coggins, J. R., C. Abell, L. B. Evans, M. Frederickson, D. A. Robinson, A. W. Roszak, and A. P. Lapthorn. 2003. Experiences with the shikimate-pathway enzymes as targets for rational drug design. Biochem. Soc. Trans. 31:548552 .

6. DeFeyter, R. C., and J. Pittard. 1986. Purification and properties of shikimate kinase II from Escherichia coli K-12. J. Bacteriol. 165:331-333.

7. Dhaliwal, B., C. E. Nichols, J. Ren, M. Lockyer, I. Charles, A. R. Hawkins, and D. K. Stammers. 2004. Crystallographic studies of shikimate binding and induced conformational changes in Mycobacterium tuberculosis shikimate kinase. FEBS Lett. 574:49-54.

8. Fleischmann, R. D., D. Alland, J. A. Eisen, L. Carpenter, O. White, J. Peterson, R. DeBoy, R. Dodson, M. Gwinn, D. Haft, E. Hickey, J. F. Kolonay, W. C. Nelson, L. A. Umayam, M. Ermolaeva, S. L. Salzberg, A. Delcher, T. Utterback, J. Weidman, H. Khouri, J. Gill, A. Mikula, W. Bishai, W. R. Jacobs, Jr., J. C. Venter, and C. M. Fraser. 2002. Whole-genome comparison of Mycobacterium tuberculosis clinical and laboratory strains. J. Bacteriol. 184:5479-5490.

9. Gerstein, M., G. Schulz, and C. Chothia. 1993. Domain closure in adenylate kinase. Joints on either side of two helices close like neighboring fingers. J. Mol. Biol. 229:494-501.

10. Gouet, P., E. Courcelle, D. I. Stuart, and F. Metoz. 1999. ESPript: analysis of multiple sequence alignments in PostScript. Bioinformatics 15:305-308

11. Graham, D. Y. 1998. Antibiotic resistance in Helicobacter pylori: implications for therapy. Gastroenterology 115:1272-1277.

12. Gu, Y., L. Reshetnikova, Y. Li, Y. Wu, H. Yan, S. Singh, and X. Ji. 2002 Crystal structure of shikimate kinase from Mycobacterium tuberculosis reveals the dynamic role of the LID domain in catalysis. J. Mol. Biol. 319:779789.

13. Herrmann, K. M., and L. M. Weaver. 1999. The shikimate pathway. Annu. Rev. Plant Physiol. Plant Mol. Biol. 50:473-503.

14. Houben, M. H., D. Van Der Beek, E. F. Hensen, A. J. Craen, E. A. Rauws, and G. N. Tytgat. 1999. A systematic review of Helicobacter pylori eradication therapy-the impact of antimicrobial resistance on eradication rates. Aliment. Pharmacol. Ther. 13:1047-1055.

15. Izhar, M., L. DeSilva, H. S. Joysey, and C. E. Hormaeche. 1990. Moderate immunodeficiency does not increase susceptibility to Salmonella typhimurium aroA live vaccines in mice. Infect. Immun. 58:2258-2261.

16. Jones, T. A., J. Y. Zou, S. W. Cowan, and M. Kjeldgaard. 1991. Improved methods for building protein models in electron density maps and the location of errors in these models. Acta Crystallogr. Sect. A 47:110-119.

17. Kraulis, P. J. 1991. MOLSCRIPT: a program to produce both detailed and schematic plots of protein structures. J. Appl. Crystallogr. 24:946-950.

18. Krell, T., J. R. Coggins, and A. J. Lapthorn. 1998. The three-dimensional structure of shikimate kinase. J. Mol. Biol. 278:983-997.

19. Krell, T., J. Maclean, D. J. Boam, A. Cooper, M. Resmini, K. Brocklehurst, S. M. Kelly, N. C. Price, A. J. Lapthorn, and J. R. Coggins. 2001. Biochemical and X-ray crystallographic studies on shikimate kinase: the important structural role of the P-loop lysine. Protein Sci. 10:1137-1149.

20. Kremer, L., and G. S. Besra. 2002. Re-emergence of tuberculosis: strategies and treatment. Expert Opin. Investig. Drugs. 11:153-157.

21. Lam, S. K., and N. J. Talley. 1998. Report of the 1997 Asia Pacific Consensus Conference on the Management of Helicobacter pylori Infection. J. Gastroenterol. Hepatol. 13:1-12.

22. Lamzin, V., and K. S. Wilson. 1993. Automated refinement of protein models. Acta Crystallogr. Sect. D 49:127-147.
23. Laskowski, R. A., M. W. MacArthur, D. S. Moss, and J. M. Thornton. 1993 PROCHECK: a program to check the stereochemical quality of protein structures. J. Appl. Crystallogr. 26:283-291.

24. Leipe, D. D., E. V. Koonin, and L. Aravind. 2003. Evolution and classification of P-loop kinases and related proteins. J. Mol. Biol. 333:781-815.

25. Lind, T., S. Veldhuyzen van Zanten, P. Unge, R. Spiller, E. Bayerdorffer, C. O'Morain, K. D. Bardhan, M. Bradette, N. Chiba, M. Wrangstadh, C. Cederberg, and J. P. Idstrom. 1996. Eradication of Helicobacter pylori using one-week triple therapies combining omeprazole with two antimicrobials: the MACH I study. Helicobacter 1:138-144.

26. Marshall, B. 2002. Helicobacter pylori: 20 years on. Clin. Med. 2:147-152.

27. McConkey, G. A. 1999. Targeting the shikimate pathway in the malaria parasite Plasmodium falciparum. Antimicrob. Agents Chemother. 43:175177.

28. Megraud, F., and H. Lamouliatte. 2003. The treatment of refractory Helicobacter pylori infection. Aliment. Pharmacol. Ther. 17:1333-1343.

29. Merrit, E. A., and M. E. P. Murphy. 1994. Raster3D version 2.0. A program for photorealistic molecular graphics. Acta Crystallogr. Sect. D 50:869-873.

30. Millar, G., A. Lewendon, M. G. Hunter, and J. R. Coggins. 1986. The cloning and expression of the aroL gene from Escherichia coli K12. Purification and complete amino acid sequence of shikimate kinase II, the aroL-gene product. Biochem. J. 237:427-437.

31. Muller-Dieckmann, H.-J., and G. E. Schulz. 1995. Substrate specificity and assembly of the catalytic center derived from two structures of ligated uridylate kinase. J. Mol. Biol. 246:522-530.

32. Murshudov, G. N., A. A. Vagin, and E. J. Dodson. 1997. Refinement of macromolecular structures by the maximum-likelihood method. Acta Crystallogr. Sect. D 53:240-255.

33. Navaza, J. 1994. AMoRe: an automated package for molecular replacement. Acta Crystallogr. Sect. A 50:157-163.

34. Otwinowski, Z., and W. Minor. 1997. Processing of X-ray diffraction data collected in oscillation mode. Methods Enzymol. 276:307-326.

35. Parsonnet, J. 1993. Helicobacter pylori and gastric cancer. Gastroenterol. Clin. N. Am. 22:89-104

36. Pereira, J. H., J. S. de Oliveira, F. Canduri, M. V. Dias, M. S. Palma, L. A. Basso, D. S. Santos, and W. F. de Azevedo, Jr. 2004. Structure of shikimate kinase from Mycobacterium tuberculosis reveals the binding of shikimic acid. Acta Crystallogr. Sect. D 60:2310-2319.

37. Ridley, R. G. 1998. Planting new targets for antiparasitic drugs. Nat. Med. 4:894-895.

38. Roberts, F., C. W. Roberts, J. J. Johnson, D. E. Kyle, T. Krell, J. R. Coggins, G. H. Coombs, W. K. Milhous, S. Tzipori, D. J. Ferguson, D. Chakrabarti, and R. McLeod. 1998. Evidence for the shikimate pathway in apicomplexan parasites. Nature 393:801-805

39. Romanowski, M. J., and S. K. Burley. 2002. Crystal structure of the Escherichia coli shikimate kinase I (AroK) that confers sensitivity to mecillinam. Proteins 47:558-562.

40. Schulz, G. E. 1992. Binding of nucleotides by proteins. Curr. Opin. Struct. Biol. 2:61-67.

41. Schulz, G. E. 1992. Induced-fit movements in adenylate kinases. Faraday Discuss. 1992:85-93

42. Schulz, G. E., C. W. Muller, and K. Diederichs. 1990. Induced-fit movements in adenylate kinases. J. Mol. Biol. 213:627-630.

43. Steinrucken, H. C., and N. Amrhein. 1980. The herbicide glyphosate is a potent inhibitor of 5-enolpyruvyl-shikimic acid-3-phosphate synthase. Biochem. Biophys. Res. Commun. 94:1207-1212.

44. Tomb, J. F., O. White, A. R. Kerlavage, R. A. Clayton, G. G. Sutton, R. D. Fleischmann, K. A. Ketchum, H. P. Klenk, S. Gill, B. A. Dougherty, K. Nelson, J. Quackenbush, L. Zhou, E. F. Kirkness, S. Peterson, B. Loftus, D. Richardson, R. Dodson, H. G. Khalak, A. Glodek, K. McKenney, L. M. Fitzegerald, N. Lee, M. D. Adams, E. K. Hickey, D. E. Berg, J. D. Gocayne, T. R. Utterback, J. D. Peterson, J. M. Kelley, M. D. Cotton, J. M. Weidman, C. Fujii, C. Bowman, L. Watthey, E. Wallin, W. S. Hayes, M. Borodovsky, P. D. Karp, H. O. Smith, C. M. Fraser, and J. C. Venter. 1997. The complete genome sequence of the gastric pathogen Helicobacter pylori. Nature 388:539-547.

45. Walker, J. E., M. Saraste, M. J. Runswick, and N. J. Gay. 1982. Distantly related sequences in the alpha- and beta-subunits of ATP synthase, myosin, kinases and other ATP-requiring enzymes and a common nucleotide binding fold. EMBO J. 1:945-951. 